\title{
IRIS - AN INNOVATIVE INSPECTION SYSTEM FOR MARITIME HULL STRUCTURES
}

\author{
M Wilken, C Cabos, DNV GL SE, Hamburg, Germany \\ D Baumbach, M Buder, A Choinowski, D Grießbach, S Zuev, DLR (German Aerospace Center), Berlin, Germany
}

\section{SUMMARY}

Today digital photographs play an important role for documenting the condition of a ship's hull structure. They are excessively taken but are typically only managed like "photos in a shoe box" with no direct link to the hull structure. This makes it difficult and sometimes impossible to localize the structure shown on the photographs for further follow up or monitoring activities if no positioning information was added manually. An automated positioning of the photos (or any other measurement) within a structural model of the vessel would resolve this problem without increasing costs for capturing photos. Within tanks, automatic positioning is difficult because of the lack of GPS signals. Therefore, during the last two years, DNV GL and DLR developed an inspection system called IRIS allowing acquisition of inspection photos, their automatic spatial referencing and an integration into a 3D model based hull integrity management system. This helmet based system takes stereo camera and inertial sensor signals as an input and allows automatic positioning of photos in a model of the ship after an inspection.

\section{MOTIVATION}

A ship's hull structure needs to be maintained in a condition sufficient to safeguard its structural integrity. To make sure that this is the case - and to initiate repair in case it is not - periodic inspections are performed.

The availability of digital cameras in the inspection and survey processes of ships has significantly increased the ability to easily document a ship's hull condition.

As an example, a typical ballast tank of a seagoing vessel is inspected once per year. This inspection is performed by either the chief mate, or, if a class survey is due, also by the respective class surveyor. As crews change regularly and also most likely a different surveyor will visit the vessel the next time, it is important that documentation of its condition is accessible and independent of person. Furthermore, it should be easy to compare the structural condition of a ship to the status during previous inspections.

Tanks or other compartments such as cargo holds on board a ship can be huge. The ballast tank of a large ore carrier can e.g. 30m high, see Fig. 1. Inspection and documentation of such tank can take a whole day.

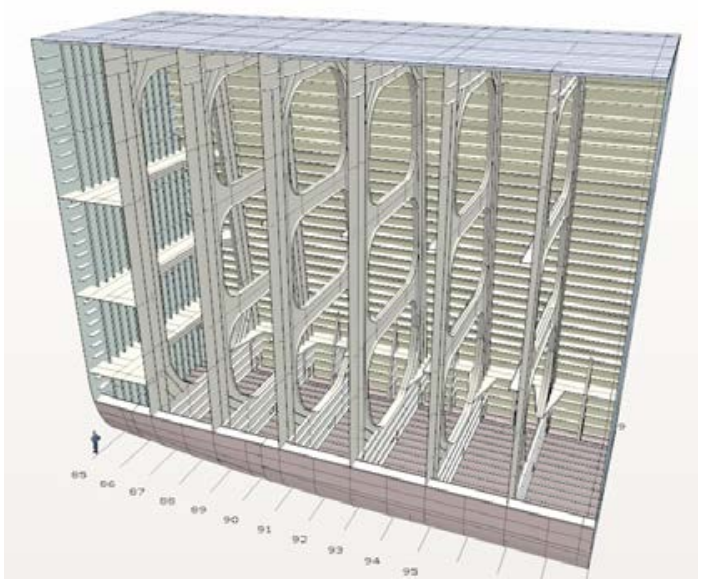

Figure 1: Ballast tank of large ore carrier with inspector drawn to scale in lower left corner.
Due to the regularity of the structure, it can be very difficult to associate any photo taken inside this tank to its actual position after the inspection.

The other extreme are narrow double bottom tanks of smaller vessels which are difficult to access because of small size openings, and difficult to navigate because of their extension, see Fig. 11. Any recordings of hull condition such as done with a camera or written notes should be attached to a specific location inside the tank to enable follow-up.

Due to the high number of photos taken and the lack of automatic mechanisms to locate such photos, the photo documentation is often stored without a reference to the structure. This can make it very hard for a person not involved in the inspection to further utilize such photos.

Inside a ship compartment, no GPS is available. Therefore, automatic localization of photos, as is customary in today's smartphones via GPS, is not possible. For that reason positioning technologies without the use of GPS has been an active area of research in recent years. Such technology would have potential benefits in many industries.

It is evident from the conditions described above, that a positioning system usable inside a ship's compartment, would increase the quality of documentation significantly if immediately applied to a photo at the moment it is taken. At the same time capturing cost could decrease as no further manual positioning needs to be performed.

Positioning can be realized with respect to different reference frames. At the most simple level, each photo would be associated with the name of the particular compartment. This is often realized today by taking a photo of a sign containing the compartment name when entering that specific place (and by assuming that all subsequent photos have been taken there).

At the next level, a simple sketch would be drawn, or a part of a drawing would be utilized to manually mark 
approximate photo positions. Alternatively, photos could be localized by manually changing their filename to a description of location.

In case a $3 \mathrm{D}$ model of the ship is available, photo documentation can be attached to the location inside the model. This functionality is available e.g. in the software system ShipManager Hull, see Fig. 2. Nevertheless, positioning of the photos is a manual process.

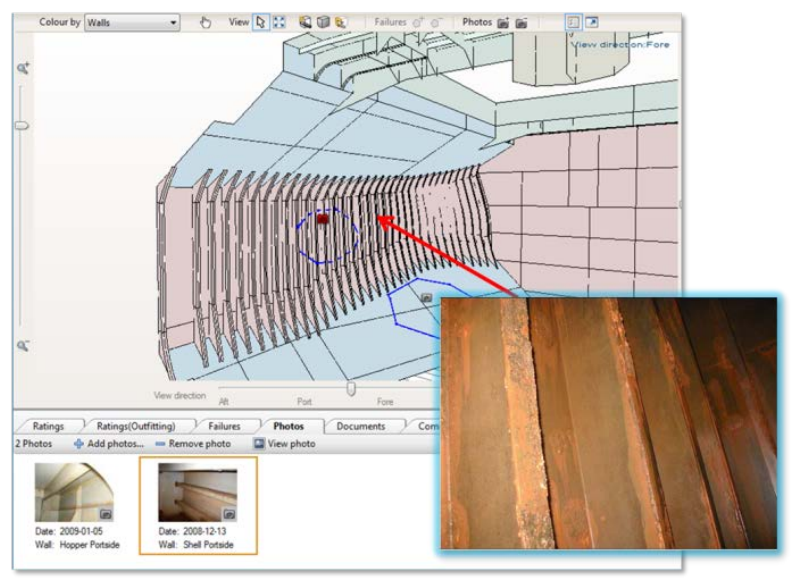

Figure 2: Positioning of photos in ShipManager Hull

In this paper the IRIS inspection system for maritime hull structures is presented which is composed of the two major components:

- The mobile positioning system IPSpect consisting of hardware components carried by inspector like helm mounted sensor head, backpack, control pen and of the firmware navigation engine for position computation (see section 2.).

- The 3D model based hull integrity software ShipManager Hull managing ship's hull condition onboard and onshore (see section 3.).

IRIS allows the automatic tracking of a camera system and orientation (position and attitude) of photos taken in rough enclosed environments such as tanks onboard ships. The system can automatically attach the photos to an existing 3D model of the tank inside the software ShipManager Hull if such model is available.

IRIS does not require the existence of such a 3D model. If the model does not exist, photos are only positioned relative to each other, without reference to the structure.

\section{POSITIONING SYSTEM}

IPSpect ${ }^{\circledR}$ (Integrated Positioning System for Inspection) developed by DLR is a mobile system dedicated to support the inspector during visual inspection of a ship's hull structure. The IPSpect system features automatic photo acquisition, storing and generation of orientation information of the photo. The recorded data (photos and corresponding orientation) are exportable into ShipManager Hull supporting the inspection process.
IPSpect consists of three main components: a) a helmet based sensor head, b) a control and power unit placed in a backpack including a control pen for taking photos and for monitoring system state and c) a software navigation engine based on visual and inertial measurements.

\section{$2.1 \quad$ HARDWARE}

The hardware setup is shown in Fig. 3 - a DLR technology demonstrator development. The sensor head is mounted to the operator helmet. This permits a handsfree inspection of the hull. The relatively small outline of the systems is also an advantage in the narrow parts of the hull.

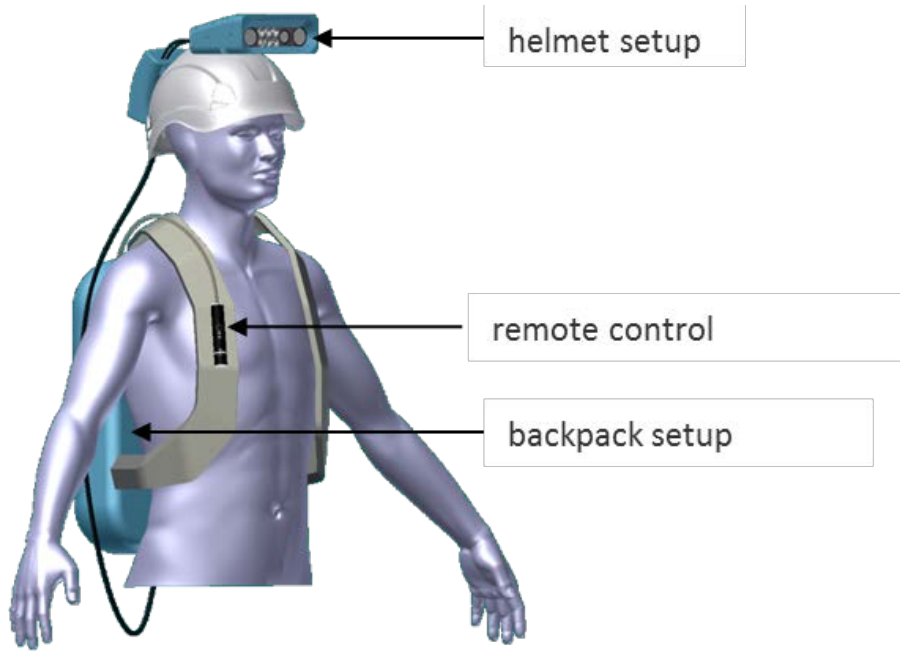

Figure 3: Integrated Positioning System for Inspection (IPSpect)

The helmet setup includes all sensors, which are needed for computing the helmet's orientation. The sensors are industrial grade cameras, an inertial measurement unit and a tilt sensor. Inspection photos are taken with a colour camera. For illumination of the camera field of view white and near-infrared LEDs are implemented. Also a diffractive optical elements laser is included for visual feedback of the field of view.

Accurate calibration and registration of all sensors is an essential prerequisite and a precise synchronization of incoming image and sensor data has to be ensured. Within IPSpect all sensors are calibrated. A fieldprogrammable gate array (FPGA) grabs the inertial sensor data and takes care of high precision data synchronization. All incoming and outbound data will be tagged with a counter value. The counter represents the local time scale to which all the sensor data is referenced to. All static latencies between the sensor data and the FPGA are calibrated.

A microcontroller allows system supervision via remote control on the backpack holder. Part of the functions are starting the recording and processing, releasing inspection pictures and showing the remaining battery life. 


\subsection{FIRMWARE NAVIGATION ENGINE}

The navigation engine runs on an embedded PC inside the backpack. The computation is done in real time during the inspection. Following the inspection on site, the images and their relative orientations may be accessed by WLAN or directly upon the data storage.

\section{2(a) Data Processing}

The software running on the embedded computer consists of different modules for data pre-processing and data processing. An overview of the dataflow is given in Fig. 4. Pre-processing (Fig. 4 middleware) has to be applied to the sensor data (e.g. protocol and format conversions). The actual data processing for the vision aided navigation, described in section 2.2(b), is applied in the application network.

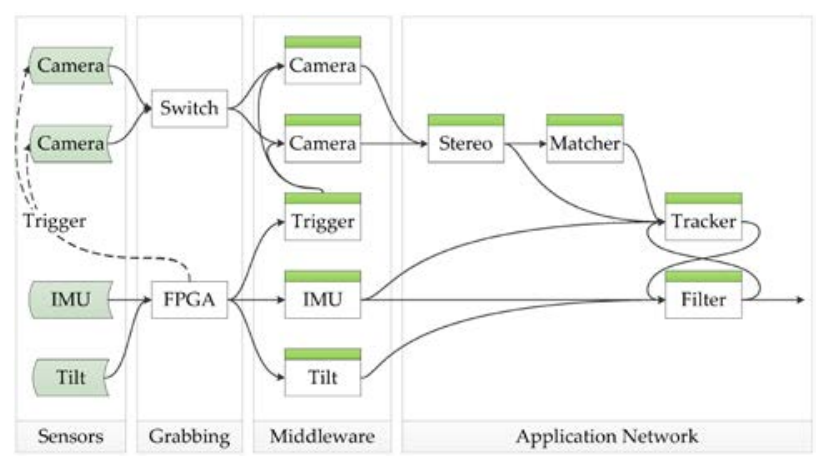

Figure 4: Data processing workflow

\section{2(b) Vision Aided Inertial Navigation}

To provide reliable and robust knowledge of position and attitude in GPS denied indoor environments, a vision aided inertial navigation system has been used [1]. This method leverages the complementary strengths of inertial and visual measurements by combining them, thereby reducing inertial sensor drift while supporting the visual system with inertial information.

Based on the dead reckoning method, an inertial navigation system calculates orientation from a known initial position, velocity, and attitude using inertial sensors - accelerometers and gyroscopes - which measure acceleration and angular velocity with respect to an inertial reference frame (see Fig. 5).

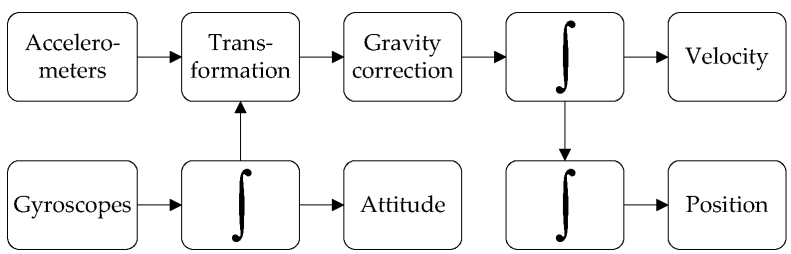

Figure 5: Mechanization of the inertial navigation system

Due to integrated errors, this leads to unbound error growth if not corrected by an independent additional measurement. Visual odometry has been proven to be able to provide such a measurement. Using a stereo system, changes in position and attitude can be estimated between two consecutive stereo image pairs from homologous image points found in those image pairs (Fig. 6). To measure in images, a precise knowledge of the geometric properties of the stereo system is needed. This is achieved by a novel camera calibration method using holographic pattern [2].

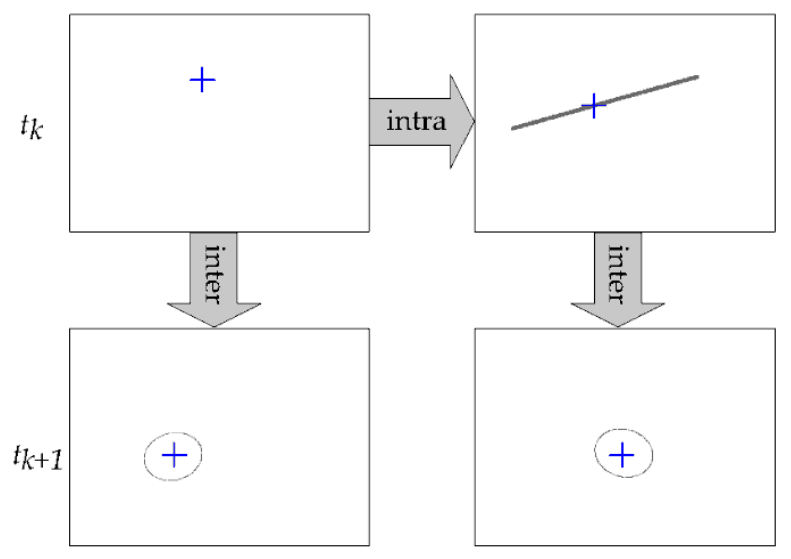

Figure 6: Stereo vision odometry

This visual measurement is fused with the inertial measurement by means of an error state Kalman filter, a recursive algorithm to determine parameters of interest from direct or indirect, noisy observations. This filter contains the errors of position, velocity, and attitude as well as the bias errors of the inertial sensors. All states are refined with each aiding measurement from the stereo system and used to correct the solution given by the inertial navigation system (Fig. 7). The output position and attitude is given in a local coordinate system aligned to a local tangent plane.

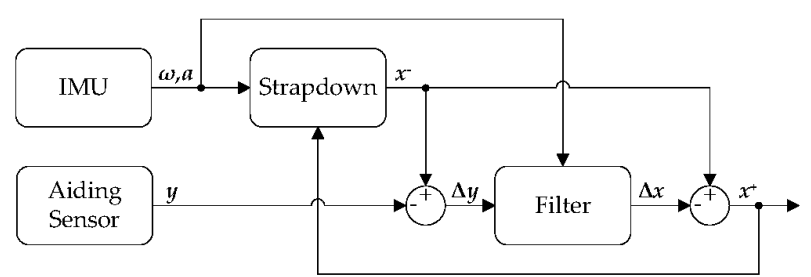

Figure 7: Error state navigation filter design

\section{HULL INTEGRITY MANAGEMENT SYSTEM}

Hull maintenance can contribute significantly to the total maintenance cost of a ship or offshore structure. As failure of the structure can have significant consequences, hull maintenance is an important part of safety management as documented by IMO International Safety Management Code (ISM), rules 10.1 and 10.2. Classification societies examine the hull structure during annual and renewal surveys to ensure that hull condition does not compromise safety.

Thorough management of the information captured during inspection and survey of the hull is beneficial both from a cost and safety perspective. Sharing of condition 
documentation such as reports, photos and measurements bears a low cost but high value if the information is sufficiently specific. Since the hull structure is large and complex, positioning of findings of all kinds is therefore crucial.

Software systems to support the hull maintenance process have gained popularity in the market since about 2010. The more advanced systems make use of a 3D model to represent the hull structure and to allow positioning of findings and follow-up actions and specification of repair. This is the case e.g. for ShipManager Hull (formerly GL HullManager), see Fig. 2 and [3] for more information. The systems which are available today require manual entry of finding information.

The mentioned software systems are typically complemented with supporting services for inspection planning, training and repair advice.

\subsection{UTILIZATION OF $\quad$ POSITONING INFORMATION}

Considering the major importance of photos for inspections and the major importance of optical sensors (cameras) of the applied positioning technology the focus of the developed IRIS system was on positioning and processing of photos in a hull integrity management system, the ShipManager Hull.

When combining a 3D model based hull integrity management system like ShipManager Hull and a positioning system like IPSpect it is crucial to consider the different coordinate systems involved. The positioning system determines 3D coordinates in a fixed, but arbitrary coordinate system whereas the hull integrity system uses the standard ship coordinate system to locate inspection data within the ship structure. Since the positioning system is not aware about its orientation in the ship coordinate system inherently, the user has to define the transformation between these two coordinate systems. The definition of this transformation is called registration and is of major importance for utilizing automated positioning data in a system having its own coordinate system. Since the positioning system is capable to retrieve the orientation of the sensor coordinate system at any points in times the trajectory of the sensor can be derived and displayed in the 3D model scenery as a simple polyline and offers a fast possibility to check the suitability of the registration.

Originally SMH knows about the spatial context and semantic of an inspection but does not know about locations of observations documented by a photo taken during an inspection run. Even though photos can be attached manually to the 3D model neither the area covered by the photo is reflected nor can positions of details shown on a photo be derived.

Using the orientation information retrieved by a positioning system the location of a photo can now be defined by the position and attitude of the inspection camera automatically. Within the SMH this is symbolized as a sphere in the $3 \mathrm{D}$ model scenery where viewing direction is indicated by a red line on the sphere. More detailed information can be displayed for a selected photo by showing the inspection photo as transparent texture of a pyramid base (see Fig. 8). The pyramid is aligned with the field of view of the inspection camera. Displaying inspection photos in this way allows an overview where the photos are taken and which structural parts are shown on the photo.

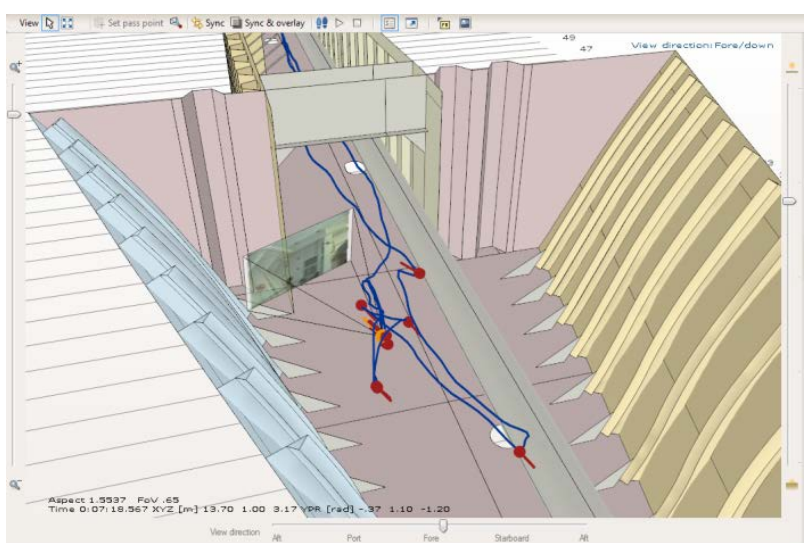

Figure 8: Helmet trajectory and inspection photos displayed in $\mathrm{SMH}$

Considering the field of view beside the position and attitude of the inspection camera, the view of the 3D model can be synchronized with the perspective of the inspection photo. This view synchronization allows a comparison between the 3D model and the real as-is condition of the hull structure and could be used for placing markups in the 3D model based on failures shown on the inspection photos. These markups can be used for later follow up activities like repair planning.

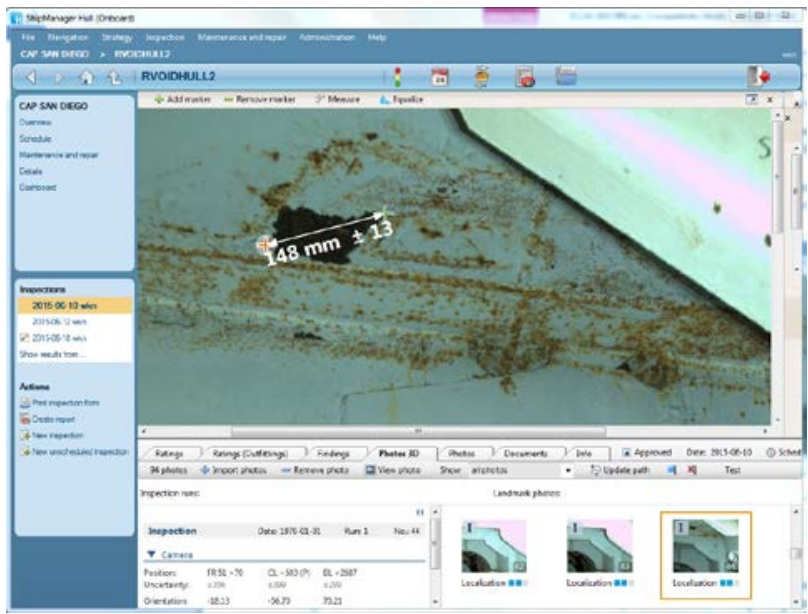

Figure 9: Measure defect in SMH by photo depth map

Furthermore a positioning system as IPSpect does not only compute position and attitude of the camera but is also able to create depth maps of the photos. This makes it possible to determine the related $3 \mathrm{D}$ coordinate of a single pixel from an inspection photo. Thus positions of depicted details can directly be retrieved from the photo. Consequently also measuring of distances in inspection photos is possible (see Fig. 9). 


\section{ADVANCED INSPECTION SYSTEM}

The developed inspection system IRIS is started by booting the tracking hardware IPSpect and awaiting a short transient phase for the sensors. Thereafter the positioning system is equipped by the inspector and the compartment to be inspected is entered. Before starting the indoor tracking recording by the control pen a calibration of the inertia sensors against the gravitational acceleration has to be performed. As a first action after starting the tracking recording the inspector has to take a photo depicting a well-known part of the structure in terms of position (e.g. a manhole) for the purpose of registration. Then the actual inspection is performed as usual and inspection photos are taken by releasing the switch of the control pen. At the end of the inspection run a further registration photo has to be taken to allow an increase in positioning accuracy later on. All recorded data are stored on the solid state disk in the backpack which is unplugged from the tracking hardware and plugged via USB cable to the computer with SMH installed. Within SMH the inspected compartment has to be selected and the tracking data (including all taken photos) have to be imported from the solid state disk subsequently. In SMH all photos can be reviewed (like standard photos) immediately. Having the registration performed (see section 4.1) all positioning information can be transformed into the ship coordinate system so that all photos are automatically placed at once at the right location in the compartment for further evaluation.

\subsection{INTERFACE POSITIONING AND HULL INTEGRITY SYSTEM}

For exchange of photo and positioning data between the IPSpect positioning system and SMH, a platform independent web service has been implemented. This allows requesting data as well as particular calculations related to the orientation of inspection photos which are needed for the correct registration of photos within the 3D ship model. Additionally a function for accurate metric measurements of objects within an inspection photo is provided. This function is based on the fusion of inspection camera photos and navigation stereo camera data.

The calculated IPSpect trajectory is relative to the starting point of the inspections session and given in local coordinate system. Therefore a coordinate transformation from local coordinates to ship coordinates, related to the 3D-Model, has to be applied. For that purpose a registration procedure is implemented, based on the identification and measuring of at least six control points with known 3D-coordinates in dedicated registration photos [4]. Having these control points available in the hull integrity model the 3D coordinates of the control points can be simply derived from it. Several of these registration locations are used to find the transformation between the local coordinate frame and the ship coordinate system. Since the accuracy of a single registration is affected by quality, distribution, and accuracy of the selected control points, at least a second registration at the end of the inspection session is designated. Using more than one registration increases the robustness und accuracy of the final absolute trajectory.

\section{ONBOARD TEST}

The developed inspection system was tested on the museum ship Cap San Diego in the port of Hamburg. The Cap San Diego was chosen as test environment due to the good continuous accessibility. Based on the available drawings a hull integrity model was set up (see Fig. 10). The shaft tunnel, pipe duct, cargo hold 1 and tank 1 were modelled with internal structure (stiffeners, web frames and manholes), all other compartments were modelled only as bounding geometry (so called Hybrid Model).

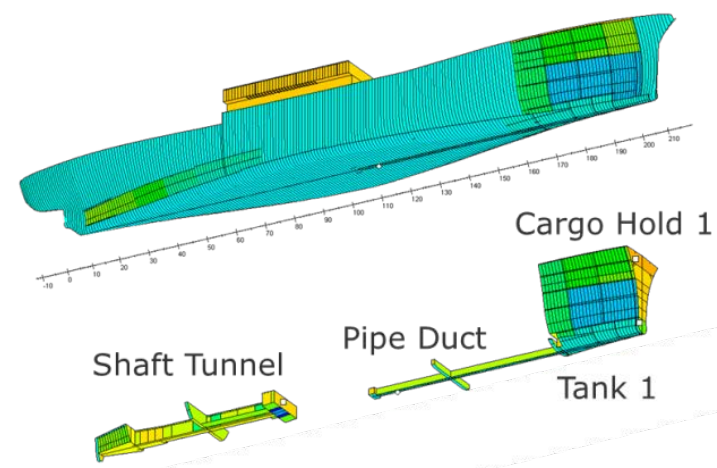

Figure 10: 3D Hull Integrity Model of Cap San Diego

The system was tested in the large cargo hold 1 , the office like shaft tunnel with good lighting and good accessibility and the dark, dirty and confined ballast water tank 1 accessible only through a man hole.

\subsection{ONBOARD USE}

The developed technology demonstrator composed of helmet mounted sensor head, the control unit carrying backpack and the control pen allowed hands free operation during the inspection. This was of major importance in particular for the very confined tank 1 (see Fig. 11). 


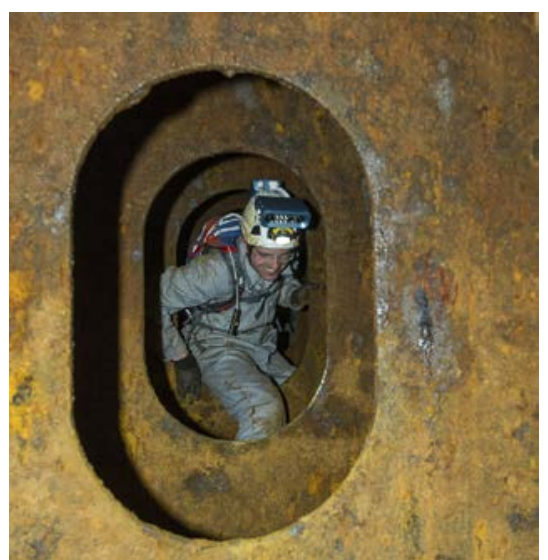

Figure 11: IPSpect in ballast water Tank 1

Nevertheless the current system should be decreased in size and weight to further improve mobility and usability for the inspector when it comes to an industrial application. With regard to the positioning capability the IPSpect was able to gather the necessary tracking sensor data for all three compartments. Even in tank 1 with difficult illumination for the stereo cameras by the selfcarried lighting (sometimes very limited, sometimes very wide view depth) and the very special movement profile of the inspector the recorded acceleration and camera data allowed automated positioning of the system.

The sensor data stored on the solid state disk of the IPSpect was plugged via USB connection to a laptop with $\mathrm{SMH}$ installed and the recorded tracking data could be directly imported and visualized in SMH onboard.

\subsection{POSITIONING}

By comparing measured with manually captured positions after the test run, the accuracy of the positioning system could be examined. As no absolute reference frame is available for the system, a certain drift builds up overtime. The computed accuracy with respect to the tracking coordinate system of the trajectories was on average $20 \mathrm{~mm} / \mathrm{min}$ in translation and $1^{\circ}$ in attitude (constant over time) for the performed test runs. This drift can be compensated retrospectively through the registration procedure.

For transforming the positioning information into the ship coordinate system the registration had to be performed. If the coordinates of the selected control points (see section 4.1) should be retrieved from the 3D model directly it had to be ensured, that the registration photo shows enough (at least 6) control points which could be clearly identified in the 3D model at the accurate position.

After consideration of the registration the trajectory and inspection photos were automatically located in the 3D model of the compartment showing a good matching of the inspection path and the 3D model (see Fig. 12).

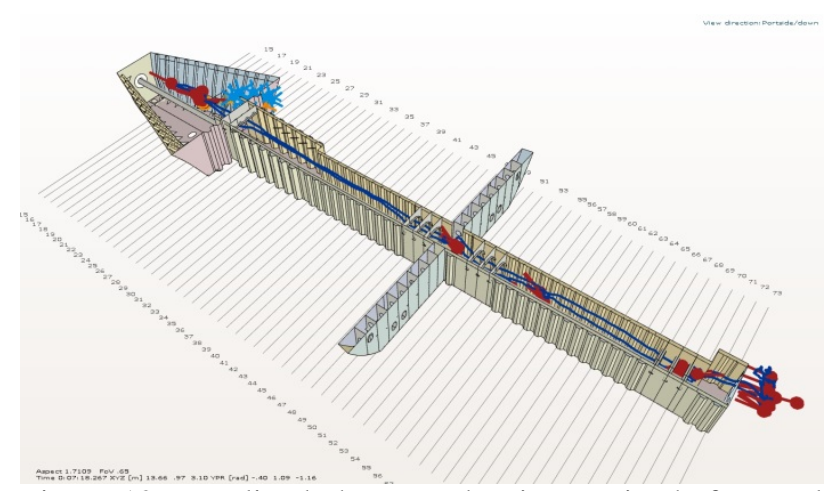

Figure 12: Localized photos and trajectory in shaft tunnel visualized in $\mathrm{SMH}$

The visual accuracy of the inspection photos was checked by application of the view synchronization between photo and 3D model scenery and found in quite good agreement: The depicted structural parts in the photo could be clearly identified in the $3 \mathrm{D}$ view of the structural model (see Fig. 13).

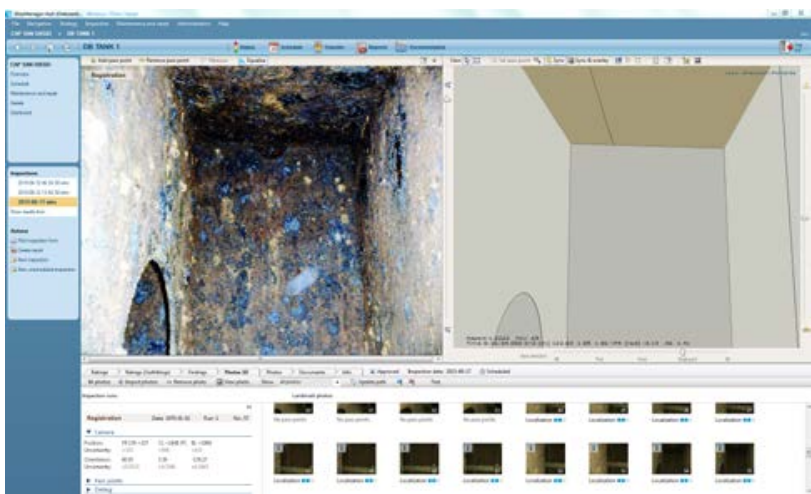

Figure 13: 3D view synchronized with photo view

\subsection{PHOTO MEASURING}

To check the accuracy of the depth maps of the inspection photos, onboard measured distances of selected structural details were compared with distances retrieved by the photo measuring functionality. The measurements were repeated by using different inspection photos showing the same structure but from slightly different perspectives.

Due to the functional principle of the applied positioning system not each pixel in an inspection photo was suited for position determination. E.g. if a pixel was part of an area showing no contrast on the photo (like a pixel of a grey wall) it was not possible to determine the position, since the pixel could not be clearly located in both stereo camera photos. This seems to be a general restriction in functionality, but typically it is not since measurements in photos are mostly required between pixels showing some features with contrast.

An example of one measuring test is shown in Fig. 14 including also one measurement, where pixel localization was not possible. 


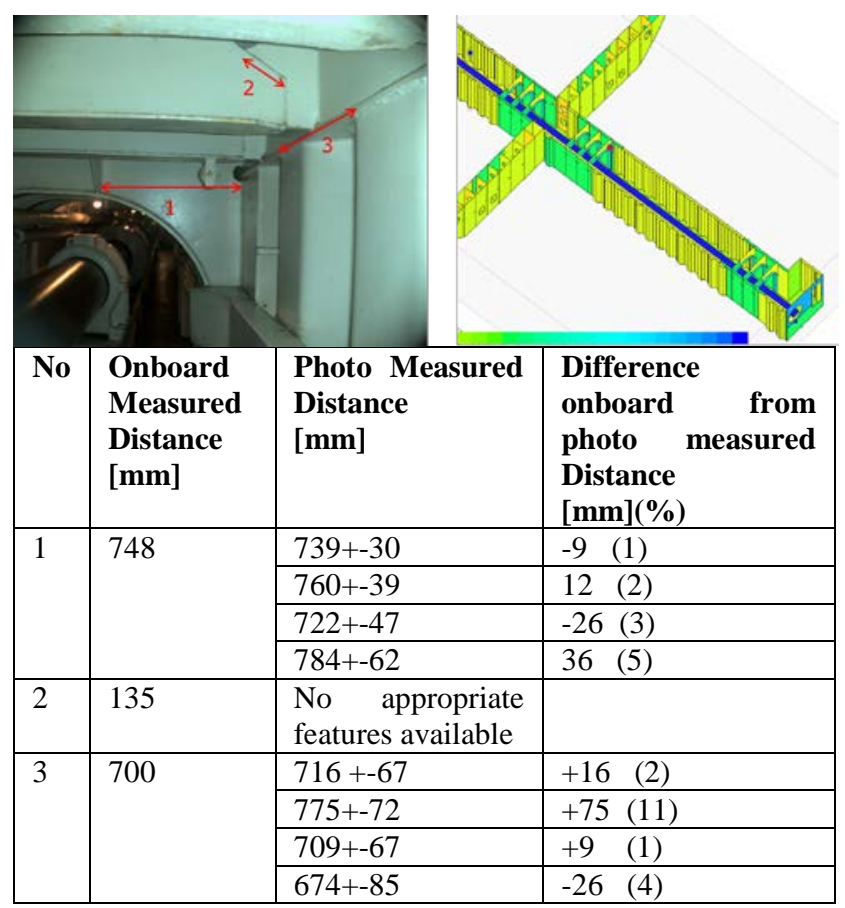

Figure 14: Distance measured in photo of shaft tunnel

Based on the results of all measurement tests made, the accuracy of measured distances in photos with respect to the onboard measured distances was on average approximately $5 \%$. These deviations are in very good agreement with the computed uncertainties from the positioning system.

\section{OUTLOOK}

The current IRIS system has the state of a functional prototype developed for proving feasibility and usefulness of joining 3D model based hull integrity management with an in-ship tracking technology. Hence focus was on demonstrating the applicability of the technology, not streamlining or fully exploitation of the technology. Based on the achieved results an outlook of possible improvements and new functionalities are given below.

\subsection{D MODEL RECONSTRUCTION}

The derivation of high density 3D point clouds from the stereo cameras of IPSpect allows improving the interpretation of the inspections results and gives the opportunity to reconstruct the real 3D model of inspected compartments. To fulfil the real time requirements the algorithms for dense stereo matching using Semi-Global matching was implemented on a graphics processor unit [5]. In combination with the navigation solution, the 3D point cloud for each image pair is transformed to the navigation frame and merged together to get a high density raw point cloud. This process is carried out offline without further data processing. Fig. 15 shows an example point cloud of the tank 1 of the Cap San Diego.
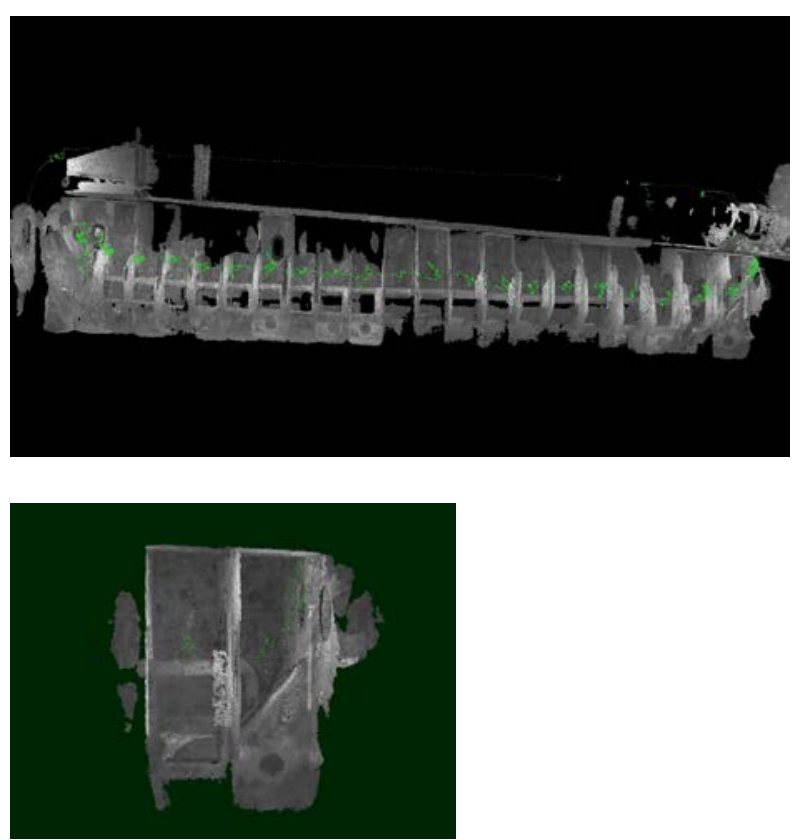

Figure 15: View of generated 3D point cloud for tank 1 of Cap San Diego (top) and for one segment (bottom)

\subsection{ADVANCES IN INSPECTION}

Indoor tracking capability is assumed as the key technology for a broad spectrum of applications, not only photo localization: Having positioning and at the same time hull information available during the inspection different further enhancements appear possible. Based on an inspection plan in $\mathrm{SMH}$, the inspector could be guided to specific areas of attention in the respective compartment. Routing within a compartment to the exit of a compartment (which is currently not always easy in confined spaces with regular internals) or provision of additional, probably historic information for an area of attention is possible.

Nevertheless also the positioned photos can be further processed. Utilizing the photo measurement functionality, repair planning can be implemented based on photos. Since general 3D coordinates can be retrieved from photos not only distances (as currently implemented) but also (repair) areas can be determined in a photo. In case of a detailed structural model plate thicknesses and material information can be determined from the $3 \mathrm{D}$ model in addition providing all relevant repair information.

With respect to hull lifecycle maintenance also the overlap in field of view of inspection photos can be computed allowing to setup up a photo history documenting the condition evolution of the structure.

\section{CONCLUSION}

IRIS, a system for an automatic positioning of photos taken during an inspection of ship compartments and tanks has been developed. It is connected to the hull inspection software ShipManager Hull which is in operational use for a large number of vessels today. 
The mobile positioning system has been tested in an onboard environment. Thereby effectivity of the procedure could be demonstrated. After the inspection with the developed system, all photos taken were referenced relative to each other spatially. Through transferring the results into the coordinate system of the compartment, all photos are attached to their location in the 3D model. The effort for this transfer is independent of the number of photos taken. This automatic procedure is therefore highly efficient and much less error-prone if a comprehensive photo documentation of the compartment condition is required.

The next development steps are tests in operational environments and industrialization of the inspection hardware.

\section{ACKNOWLEDGEMENTS}

The authors gratefully acknowledge Ms. Liedtke from DNV GL for the professional implementation of positioning information in ShipManager Hull, the crew of Cap San Diego in Hamburg for the support during onboard tests and Ms. Ines Ernst from DLR for generating of 3D point clouds from onboard campaign.

\section{REFERENCES}

1. GRIESSBACH, D.; BAUMBACH, D., ZUEV, S., "Stereo vision aided inertial navigation for unknown indoor and outdoor environments", The Fifth International Conference on Indoor Positioning and Indoor Navigation 2014 (IPIN 2014), 2014

2. M. BAUER, D. GRIESSBACH, A. HERMERSCHMIDT, S. KRÜGER, M. SCHEELE, AND A. SCHIMSCHMANOW, "Geometrical camera calibration with diffractive optical elements", Opt. Express 16, 20241-20248, 2008

3. LANGBECKER, U., CABOS, C., 'Adoption of 3d Hull Maintenance Software in Ship Operation', Proceedings of ICCAS, Trieste, 2011.

4. V. LEPETIT, F. MORENO-NOGUER AND P. FUA., "EPnP: An Accurate O(n) Solution to the PnP Problem”, International Journal Of Computer Vision, vol. 81, p. 155-166, 2009.

5. ERNST, I. AND HIRSCHMÜLLER, H., "Mutual Information Based Semi-Global Stereo Matching on the GPU”, In: ISVC '08: Proceedings of the 4th International Symposium on Advances in Visual Computing, Springer-Verlag, Berlin, Heidelberg, pp. 228-239., 2008

\section{AUTHORS BIOGRAPHY}

Christian Cabos is responsible for Information Management Technologies in DNV GL Maritime
Technology and R\&D. This includes the development of new computer-based methods in the fields of maintenance, data exchange, PLM and computational mechanics. His professional background is in numerical mathematics and physics.

Marc Wilken is deputy head of section Information Management R\&D in DNV GL where he is responsible for the development of inspection technologies and computational engineering tools for vibration, acoustics and strength. His professional background is physics.

Dirk Baumbach is scientific associate at DLR, Institute of Optical Sensor Systems department of Information Processing of Optical Systems. His research interests involve inertial navigation, sensor fusion and calibration.

Maximilian Buder is responsible for the hardware engineering, firmware development and hardware and software tests at DLR, Institute of Optical Sensor Systems. His professional background is computer science.

André Choinowski is responsible for the demonstrator hardware engineering, firmware development, hardware and software tests and measurement campaign realization at DLR, Institute of Optical Sensor Systems. His professional background is IT and product development.

Denis Grießbach is responsible for the inertial vision aided navigation algorithms as well as the web interface integration at DLR, Institute of Optical Sensor Systems. His research is concerned with computer vision and inertial navigation.

Sergey Zuev is responsible for multi sensor navigation team at DLR Institute of Optical Sensor Systems. This includes research and development of new technologies in positioning and navigation. His professional background is in control systems. 